\title{
INFLUÊNCIA DE FATORES EPIDEMIOLÓGICOS NO SEGUIMENTO E APARECIMENTO DE PROBLEMAS PUERPERAIS
}

\author{
INFLUENCE OF EPIDEMIOLOGICAL FACTORS \\ ON FOLLOW-UP AND APPEARANCE OF \\ PUERPERAL PROBLEMS
}

\section{INFLUENCIA DE FACTORES EPIDEMIOLÓGICOS EN EL SEGUIMIENTO Y LA APARICIÓN DE PROBLEMAS PUERPERALES}

\author{
Isabela Cristina Beskow Oliveira ${ }^{1}$ \\ Rosane Meire Munhak da Silva ${ }^{2}$ \\ Helder Ferreira ${ }^{3}$ \\ Rosangela Aparecida Pimenta Ferrari ${ }^{4}$ \\ Adriana Zilly ${ }^{5}$
}

Como citar este artigo: Oliveira ICB, Silva RMM, Ferreira H, Ferrari RAP, Zilly A. Influência de fatores epidemiológicos no seguimento e aparecimento de problemas puerperais. Rev baiana enferm. 2020;34:e35763.

\begin{abstract}
Objetivo: descrever a influência de fatores epidemiológicos para o seguimento puerperal e para o aparecimento de problemas de saúde em região coberta pela Rede Mãe Paranaense. Método: pesquisa descritiva, quantitativa, com 280 puérperas de municípios da nona Regional de Saúde do Paraná, realizada no segundo semestre de 2017 e primeiro de 2018, organizada em duas etapas: no alojamento conjunto de maternidades e nos domicílios, após seis meses do parto. Resultados: a consulta na primeira semana após o parto foi realizada por $51,1 \%$ das puérperas, e com 42 dias $76,8 \%$. Baixa idade e escolaridade foram associadas a não realização das consultas. Identificou-se $20,7 \%$ de puérperas com problemas clínicos, 53,2\% com sintomas emocionais, e aproximadamente 10\% com sintomas depressivos e pensamentos suicidas. Conclusão: fatores epidemiológicos têm influência para a adesão insatisfatória de consultas puerperais, mas não para o aparecimento de problemas de saúde no puerpério.
\end{abstract}

Descritores: Período Pós-parto. Fatores Epidemiológicos. Saúde da Mulher. Cuidados de Enfermagem.

Objective: to describe the influence of epidemiological factors on the puerperal follow-up and on the emergence of health problems in the region covered by the Mãe Paranaense Network. Method: descriptive, quantitative research, with 280 puerperal women from counties of the ninth Health Region of Paraná, held in the second half of 2017 and first half of 2018, organized in two steps: in the rooming-in care of maternities and in the homes, six months after the birth. Results: the appointment in the first week after delivery was performed by $51.1 \%$ of the puerperal women, and with 42 days, by $76.8 \%$. Low age and schooling were associated with the non-completion of appointments. There were 20.7\% of puerperal women with clinical problems, 53.2\% with emotional symptoms, and approximately 10\%

Enfermeira. Pesquisadora independente. Foz do Iguaçu, Paraná, Brasil. http://orcid.org/0000-0002-2482-654X.

Enfermeira. Doutora em Ciências. Professora Adjunta da Universidade Estadual do Oeste do Paraná. Foz do Iguaçu, Paraná, Brasil. zanem20 I0@hotmail.com. http:// orcid.org/0000-0003-3355-0132

3 Enfermeiro. Doutor em Ciências. Professor Adjunto da Universidade Estadual do Oeste do Paraná. Foz do Iguaçu, Paraná, Brasil. http://orcid.org/0000-0003-07। 58057.

Enfermeira. Doutora em Ciências. Professora Adjunta da Universidade Estadual de Londrina. Londrina, Paraná, Brasil. http://orcid.org/0000-0003-0157-746।.

Bióloga. Doutora em Ciências. Professora Associada da Universidade Estadual do Oeste do Paraná. Foz do Iguaçu, Paraná, Brasil. http://orcid.org/0000-0002-87|48205. 
with depressive symptoms and suicidal thoughts. Conclusion: epidemiological factors influence the poor adherence to puerperal appointments, but not the emergence of health problems in the puerperium.

Descriptors: Postpartum Period. Epidemiologic Factors. Women's Health. Nursing Care.

Objetivo: describir la influencia de los factores epidemiológicos para el seguimiento puerperal y la aparición de problemas de salud en la región cubierta por la Red Mãe Paranaense. Método: estudio descriptivo, cuantitativo, con 280 puérperas de municipios de la novena Salud Regional de Paraná, que tuvo lugar en la segunda mitad de 2017 y primera mitad de 2018, organizada en dos pasos: en el alojamiento conjunto de las maternidades y en los hogares, seis meses después del nacimiento. Resultados: la consulta en la primera semana después del parto fue realizada por el 51,1\% de las puérperas, y con 42 dias, por el 76,8\%. Baja edad y escolaridad se asociaron con la no realización de consultas. Se identificó el 20,7\% de puérperas con problemas clínicos, el 53,2\% con síntomas emocionales, y aproximadamente el 10\% con sintomas de depresión y pensamientos suicidas. Conclusión: factores epidemiológicos influyen en la adherencia deficiente de consultas puerperales, pero no en la aparición de problemas de salud en el puerperio.

Descriptores: Período Posparto. Factores Epidemiológicos. Salud de la Mujer. Atención de Enfermería.

\section{Introdução}

O período pós-parto, também conhecido por puerpério, tem início após a dequitação placentária e termina quando o organismo da mulher retorna às condições pré-gravídicas, por volta de seis semanas depois do parto. É considerado um momento de transição importante para a mulher, com intensas transformações em dimensões biológicas, psicológicas, comportamentais e socioculturais ${ }^{(1)}$.

Essas transformações, isoladas ou agrupadas, podem resultar em vulnerabilidades para as mulheres que vivenciam o pós-parto. Desse modo, no puerpério, essas mulheres precisam ser atendidas em sua totalidade, por meio de uma visão integral que contemple todas as suas dimensões, tendo em vista que muitos aspectos podem prejudicar sua saúde física e mental, assim como podem interferir na atenção à sua saúde nesse período de fragilidade ${ }^{(2-3)}$.

No contexto das políticas de acompanhamento do Brasil, os aspectos referidos geralmente envolvem baixa idade materna, escolaridade e renda, situação conjugal comprometida, falta de apoio familiar, cultura, paridade, seguimento inadequado no pré-natal, problemas de saúde prévios, e a falta de acesso aos serviços de saúde, entre outros ${ }^{(4-5)}$. Um estudo americano identificou que idade materna acima de 35 anos, etnia negra, histórico de problemas de saúde prévio elevam consideravelmente o risco de mulheres apresentarem complicações no pós-parto com necessidade de reinternações hospitalares ${ }^{(6)}$.

Segundo o Ministério da Saúde (MS) do Brasil, os profissionais de saúde precisam estar atentos e preparados para atender às necessidades e aos aspectos que fragilizam a saúde materna e infantil, bem como devem aproveitar a oportunidade de contato com a mulher para viabilizar ações que compõem a "Primeira Semana de Saúde Integral". Nesse contato, além dos cuidados com o recém-nascido, pode ser feita uma análise sobre a gestação e o parto, se houve intercorrências, como ocorreu o atendimento, uso de medicações, aleitamento materno, dores, fluxo vaginal/sangramentos, alimentação, sono, atividades físicas, planejamento familiar, condições psicoemocionais e sociais ${ }^{(7)}$.

A recomendação do Ministério da Saúde, assim como da Rede Mãe Paranaense (RMP), política de atenção à saúde materna e infantil, vigente no estado do Paraná desde 2012, envolve uma visita domiciliar na primeira semana após a alta do bebê, preferencialmente até o quinto dia de vida, e o retorno da mulher e do recém-nascido ao serviço de saúde, para uma consulta médica ou de enfermagem, com 42 dias após o parto ${ }^{(7-8)}$.

Dada a importância do seguimento puerperal, considerando as fragilidades presentes nessa fase importante da vida de uma mulher 
e a necessidade de práticas qualificadas e oportunas para promover a saúde materna e prevenir agravos, este estudo tem como objetivo descrever a influência de fatores epidemiológicos para o seguimento puerperal e para o aparecimento de problemas de saúde em região coberta pela RMP.

\section{Método}

Pesquisa descritiva, de corte transversal e abordagem quantitativa, realizada em maternidades de municípios pertencentes à nona Regional de Saúde do Estado do Paraná, selecionados por meio de sorteio: Foz do Iguaçu, Santa Terezinha de Itaipu, Medianeira, Serranópolis do Iguaçu, Matelândia, São Miguel do Iguaçu, Missal, Itaipulândia e Ramilândia, todos com cobertura da RMP.

Participaram da pesquisa puérperas submetidas a partos normais e cesarianas com recém-nascidos vivos em municípios pertencentes à nona Regional de Saúde. Excluíram-se as puérperas com problemas de saúde mental descrito em prontuário e mulheres com impossibilidade da visita domiciliar após três tentativas, por não encontrar o endereço, por mudança de telefone ou por estarem ausentes no momento da visita.

O cálculo amostral foi realizado com base no número de partos do ano de 2015, considerando $\mathrm{N}$ tamanho (número de elementos) da população; n tamanho (número de elementos) da amostra; $n^{0}$ uma primeira aproximação para o tamanho da amostra; $\mathrm{E}_{0}$ erro amostral tolerável ${ }^{(9)}$. Por conhecer o tamanho da população realizou-se o cálculo amostral na seguinte expressão:

$$
\mathrm{n}^{0}=1 /\left(\mathrm{E}_{0}\right)^{2} \cdot 0,05=400
$$

Conhecendo o tamanho da população, a fórmula do cálculo anterior foi substituída por:

$$
\mathrm{n}=\mathrm{N} \cdot \mathrm{n}^{0} / \mathrm{N}+\mathrm{n}_{0}
$$

Considerou-se uma margem de erro da pesquisa de 5\% e um nível de confiança de 95\%. Definiu-se 10\% como margem de segurança; no entanto, tendo em vista que este estudo foi de seguimento, ocorreram perdas maiores ao longo do período de coleta de dados, por mudança de endereço ou número telefônico. Na primeira etapa da pesquisa participaram 397 puérperas e na segunda, 280, totalizando uma perda de 29,47\% das participantes.

A coleta de dados foi organizada em duas etapas distintas, no segundo semestre de 2017 e no primeiro semestre de 2018. A primeira etapa aconteceu em três maternidades, onde foram apresentados os objetivos da pesquisa e coletado a assinatura no Termo de Consentimento Livre e Esclarecido (TCLE), realizando-se em seguida, uma breve caracterização das participantes.

As instituições hospitalares envolvidas foram:

a) Foz do Iguaçu, referência para todas as gestantes do município e para Santa Terezinha de Itaipu, independentemente do risco gestacional, e também referência para gestação de alto risco para os nove municípios que compõem a nona regional de saúde;

b) Medianeira, referência para gestantes de risco habitual para o município e para Serranópolis do Iguaçu;

c) Matelândia, referência para gestantes de risco habitual para o município e para Ramilândia.

Para a segunda etapa, realizou-se uma visita domiciliar, aproximadamente seis meses após o parto, para buscar informações a respeito do seguimento puerperal e complicações apresentadas nesse período. Para tanto, utilizou-se um instrumento estruturado com as seguintes variáveis: consulta puerperal na primeira semana pós-parto (sim ou não); consulta puerperal (sim ou não); problemas de saúde (sim ou não); condições emocionais presentes (calma, nervosa, ansiosa, depressiva) e sintomas emocionais (falta de interesse em si própria, perda de prazer, falta de energia e motivação, sentimentos de inutilidade e culpa, alterações no apetite ou peso, pensamentos recorrentes de morte ou suicídio, dormir mais ou menos do que o habitual, sentimentos negativos para com o bebê, se achou que não iria dar conta de cuidar do bebê; sentimentos de incompetência, falta de interesse no bebê, se chorava sem motivo, outros).

A coleta de dados foi realizada por acadêmicos do quarto e quinto ano do curso de graduação em enfermagem de uma instituição 
pública, os quais receberam treinamento prévio para a busca das informações.

Os dados foram analisados por meio do teste $\chi 2$ e Teste $G$, visando determinar a possível diferença, sendo adotado um nível de significância de 5\%. A análise dos dados foi realizada utilizando o programa Statistica 8.0.

A pesquisa foi submetida e aprovada pelo Comitê de Ética em Pesquisa (CEP) da Universidade Estadual de Londrina, sob o Parecer n. 2.053.304, CAAE: 67574517.1.1001.5231, e atendeu às normas da Resolução n. 466/2012, que envolve pesquisa com seres humanos.

\section{Resultados}

Os resultados mostraram que 51,1\% das 280 mulheres participantes da pesquisa realizaram a consulta puerperal na primeira semana após o parto. A Tabela 1 mostra fatores que podem influenciar a realização dessa consulta, e, pelas análises estatísticas, não foram observadas diferenças significantes que pudessem favorecer ou não a realização de tal consulta na primeira semana após o parto.

Tabela 1 - Relação dos fatores epidemiológicos com a realização ou não da consulta puerperal na primeira semana após o parto. Foz do Iguaçu, Paraná, Brasil - 2018 (N=280)

(continua)

\begin{tabular}{|c|c|c|c|c|c|}
\hline \multirow{2}{*}{ Variáveis } & \multicolumn{2}{|c|}{ Consulta $1^{\mathrm{a}}$ semana } & \multicolumn{2}{|c|}{ Sem consulta $1^{\text {a }}$ semana } & \multirow{2}{*}{ Valor de $p$} \\
\hline & $\mathbf{n}$ & $\%$ & $\mathbf{n}$ & $\%$ & \\
\hline Idade & & & & & $0,0746^{*}$ \\
\hline$\leq 19$ anos & 16 & 11,19 & 29 & 21,17 & \\
\hline 20 a 34 anos & 106 & 74,13 & 91 & 66,42 & \\
\hline$\geq 35$ anos & 21 & 14,69 & 17 & 12,41 & \\
\hline Raça/cor & & & & & $0,8309^{* *}$ \\
\hline Branca & 77 & 53,85 & 75 & 54,74 & \\
\hline Negra & 12 & 8,39 & 10 & 7,30 & \\
\hline Parda & 52 & 36,36 & 51 & 37,23 & \\
\hline Outras & 1 & 0,70 & 1 & 0,73 & \\
\hline Sem registro & 1 & 0,70 & 0 & 0,00 & \\
\hline \multicolumn{6}{|l|}{ Escolaridade } \\
\hline Fundamental completo & 14 & 9,79 & 8 & 5,84 & $0,0721 * *$ \\
\hline Fundamental incompleto & 26 & 18,18 & 34 & 24,82 & \\
\hline Médio completo & 55 & 38,46 & 58 & 42,34 & \\
\hline Médio incompleto & 22 & 15,38 & 28 & 20,44 & \\
\hline Superior completo & 15 & 10,49 & 5 & 3,65 & \\
\hline Superior incompleto & 9 & 6,29 & 3 & 2,19 & \\
\hline Sem escolaridade & 2 & 1,40 & 1 & 0,73 & \\
\hline \multicolumn{6}{|l|}{ Situação conjugal } \\
\hline Com companheiro & 132 & 92,31 & 125 & 91,24 & $0,7452^{*}$ \\
\hline Sem companheiro & 11 & 7,69 & 12 & 8,76 & \\
\hline \multicolumn{6}{|l|}{ Tem outros filhos } \\
\hline Sim & 88 & 61,54 & 74 & 54,01 & $0,2025^{*}$ \\
\hline Não & 55 & 38,46 & 63 & 45,99 & \\
\hline \multicolumn{6}{|l|}{ Ocupação materna } \\
\hline Remunerada & 74 & 51,75 & 59 & 43,07 & $0,1820 *$ \\
\hline Não remunerada & 69 & 48,25 & 78 & 56,93 & \\
\hline \multicolumn{6}{|l|}{ Renda familiar } \\
\hline Menos que 1 salário mínimo & 32 & 22,38 & 21 & 15,33 & $0,1831^{* *}$ \\
\hline 1 a 2 salários mínimos & 62 & 43,36 & 64 & 46,72 & \\
\hline 2 a 3 salários mínimos & 31 & 21,68 & 35 & 25,55 & \\
\hline Mais que 3 salários mínimos & 17 & 11,89 & 12 & 8,76 & \\
\hline Não informou & 1 & 0,70 & 5 & 3,65 & \\
\hline
\end{tabular}


Tabela 1 - Relação dos fatores epidemiológicos com a realização ou não da consulta puerperal na primeira semana após o parto. Foz do Iguaçu, Paraná, Brasil - 2018 (N=280)

(conclusão)

\begin{tabular}{|c|c|c|c|c|c|}
\hline \multirow{2}{*}{ Variáveis } & \multicolumn{2}{|c|}{ Consulta $1^{\mathrm{a}}$ semana } & \multicolumn{2}{|c|}{ Sem consulta $1^{\text {a }}$ semana } & \multirow{2}{*}{ Valor de $p$} \\
\hline & $\mathbf{n}$ & $\%$ & $\mathbf{n}$ & $\%$ & \\
\hline \multicolumn{6}{|c|}{ Bolsa família } \\
\hline Sim & 22 & 15,38 & 22 & 16,06 & $0,8769 *$ \\
\hline Não & 121 & 84,62 & 115 & 83,94 & \\
\hline
\end{tabular}

Fonte: Elaboração própria.

*Teste Qui-quadrado. **Teste G.

Embora não observado significância estatística, fatores como baixa idade, escolaridade e renda, assim como não ter outros filhos e não ter uma profissão remunerada estiveram mais presentes entre mulheres que não realizaram o seguimento puerperal na primeira semana.
No tocante à realização da consulta puerperal no período de 42 dias após o parto, verificou-se que $76,8 \%$ das mulheres realizaram a consulta e que fatores epidemiológicos podem fragilizar a continuidade do cuidado puerperal (Tabela 2).

Tabela 2 - Relação entre fatores epidemiológicos e a realização da consulta puerperal no período de 42 dias após o parto. Foz do Iguaçu, Paraná, Brasil - 2018 (N=280)

(continua)

\begin{tabular}{|c|c|c|c|c|c|}
\hline \multirow{2}{*}{ Variáveis } & \multicolumn{2}{|c|}{ Consulta puerperal } & \multicolumn{2}{|c|}{ Sem consulta puerperal } & \multirow{2}{*}{ Valor de $p$} \\
\hline & $\mathbf{n}$ & $\%$ & $\mathbf{n}$ & $\%$ & \\
\hline Idade & & & & & $0,0007^{*}$ \\
\hline$\leq 19$ anos & 25 & 11,63 & 20 & 30,77 & \\
\hline 20 a 34 anos & 157 & 73,02 & 40 & 61,54 & \\
\hline$\geq 35$ anos & 33 & 15,35 & 5 & 7,69 & \\
\hline Raça/cor & & & & & $0,6973^{* *}$ \\
\hline Branca & 119 & 55,35 & 33 & 50,77 & \\
\hline Negra & 16 & 7,44 & 6 & 9,23 & \\
\hline Parda & 77 & 35,81 & 26 & 40,00 & \\
\hline Outras & 2 & 0,93 & 0 & 0,00 & \\
\hline Sem registro & 1 & 0,47 & 0 & 0,00 & \\
\hline Escolaridade & & & & & $0,0022^{* *}$ \\
\hline Fundamental completo & 19 & 8,84 & 3 & 4,62 & \\
\hline Fundamental incompleto & 40 & 18,60 & 20 & 30,77 & \\
\hline Médio completo & 88 & 40,93 & 25 & 38,46 & \\
\hline Médio incompleto & 34 & 15,81 & 16 & 24,62 & \\
\hline Superior completo & 3 & 1,40 & 0 & 0,00 & \\
\hline Superior incompleto & 20 & 9,30 & 0 & 0,00 & \\
\hline Sem escolaridade & 11 & 5,12 & 1 & 1,54 & \\
\hline Situação conjugal & & & & & $0,4899^{*}$ \\
\hline Com companheiro & 196 & 91,16 & 61 & 93,85 & \\
\hline Sem companheiro & 19 & 8,84 & 4 & 6,15 & \\
\hline Tem outros filhos & & & & & $0,4548^{*}$ \\
\hline Sim & 88 & 40,93 & 30 & 46,15 & \\
\hline Não & 127 & 59,07 & 35 & 53,85 & \\
\hline Ocupação materna & & & & & $0,0959 *$ \\
\hline Remunerada & 108 & 50,23 & 25 & 38,46 & \\
\hline Não remunerada & 107 & 49,77 & 40 & 61,54 & \\
\hline
\end{tabular}


Tabela 2 - Relação entre fatores epidemiológicos e a realização da consulta puerperal no período de 42 dias após o parto. Foz do Iguaçu, Paraná, Brasil - 2018 (N=280)

\begin{tabular}{|c|c|c|c|c|c|}
\hline \multirow{2}{*}{ Variáveis } & \multicolumn{2}{|c|}{ Consulta puerperal } & \multicolumn{2}{|c|}{ Sem consulta puerperal } & \multirow{2}{*}{ Valor de $p$} \\
\hline & $\mathbf{n}$ & $\%$ & $\mathbf{n}$ & $\%$ & \\
\hline Renda familiar & & & & & $0,0726^{* *}$ \\
\hline $\begin{array}{l}\text { Menos que } 1 \text { salário } \\
\text { mínimo }\end{array}$ & 37 & 17,21 & 16 & 24,62 & \\
\hline 1 a 2 salários mínimos & 100 & 46,51 & 26 & 40,00 & \\
\hline 2 a 3 salários mínimos & 51 & 23,72 & 15 & 23,08 & \\
\hline $\begin{array}{l}\text { mais que } 3 \text { salários } \\
\text { mínimos }\end{array}$ & 25 & 11,63 & 4 & 6,15 & \\
\hline não informou & 2 & 0,93 & 4 & 6,15 & \\
\hline \multicolumn{6}{|l|}{ Bolsa família } \\
\hline Sim & 31 & 14,42 & 13 & 20,00 & $0,2786^{*}$ \\
\hline Não & 184 & 85,58 & 52 & 80,00 & \\
\hline
\end{tabular}

Fonte: Elaboração própria.

*Teste Qui-quadrado. ${ }^{* *}$ Teste G.

Verificou-se que existe diferença significativa entre escolaridade e a realização da consulta puerperal. A análise de resíduos mostrou que as mães com ensino fundamental incompleto foram as que menos realizaram a consulta, e as mães com ensino superior incompleto foram as que mais realizaram as consultas puerperais. A análise de resíduos ajustados mostrou que a maior diferença observada foi para as mães com idade inferior a 19 anos, sendo que a maioria não realizou a consulta.

No período puerperal podem aparecer problemas de saúde física e psicológica. Desse modo, torna-se indispensável o seguimento à saúde da puérpera. Identificou-se que 20,7\% das mulheres apresentaram problemas clínicos, e conforme mostra a Tabela 3, não foram encontradas relações estatísticas significativas entre fatores epidemiológicos e maiores chances de agravos de saúde.

Tabela 3 - Relação de fatores epidemiológicos com o aparecimento de problemas clínicos no período puerperal. Foz do Iguaçu, Paraná, Brasil - 2018 (N=280)

(continua)

\begin{tabular}{|c|c|c|c|c|c|}
\hline \multirow{2}{*}{ Variáveis } & \multicolumn{2}{|c|}{ Problemas clínicos } & \multicolumn{2}{|c|}{ Sem problemas clínicos } & \multirow{2}{*}{ Valor de $p$} \\
\hline & $\mathbf{n}$ & $\%$ & $\mathbf{n}$ & $\%$ & \\
\hline Idade & & & & & $0,2008^{*}$ \\
\hline$\leq 19$ anos & 8 & 13,79 & 37 & 16,67 & \\
\hline 20 a 34 anos & 38 & 65,52 & 159 & 71,62 & \\
\hline$\geq 35$ anos & 12 & 20,69 & 26 & 11,71 & \\
\hline Raça/cor & & & & & $0,1194^{* *}$ \\
\hline Branca & 35 & 60,34 & 117 & 52,70 & \\
\hline Negra & 2 & 3,45 & 20 & 9,01 & \\
\hline Parda & 19 & 32,76 & 84 & 37,84 & \\
\hline Outras & 2 & 3,45 & 0 & 0,00 & \\
\hline Sem registro & 0 & 0,00 & 1 & 0,45 & \\
\hline Escolaridade & & & & & $0,2845^{* *}$ \\
\hline Fundamental completo & 2 & 3,45 & 20 & 9,01 & \\
\hline Fundamental incompleto & 13 & 22,41 & 47 & 21,17 & \\
\hline Médio completo & 24 & 41,38 & 89 & 40,09 & \\
\hline Médio incompleto & 8 & 13,79 & 42 & 18,92 & \\
\hline Superior completo & 0 & 0,00 & 3 & 1,35 & \\
\hline Superior incompleto & 6 & 10,34 & 14 & 6,31 & \\
\hline Sem escolaridade & 5 & 8,62 & 7 & 3,15 & \\
\hline Situação conjugal & & & & & $0,8998^{* *}$ \\
\hline Com companheiro & 53 & 91,38 & 204 & 91,89 & \\
\hline Sem companheiro & 5 & 8,62 & 18 & 8,11 & \\
\hline
\end{tabular}


Tabela 3 - Relação de fatores epidemiológicos com o aparecimento de problemas clínicos no período puerperal. Foz do Iguaçu, Paraná, Brasil - 2018 (N=280)

\begin{tabular}{|c|c|c|c|c|c|}
\hline \multirow{2}{*}{ Variáveis } & \multicolumn{2}{|c|}{ Problemas clínicos } & \multicolumn{2}{|c|}{ Sem problemas clínicos } & \multirow{2}{*}{ Valor de $p$} \\
\hline & $\mathbf{n}$ & $\%$ & $\mathbf{n}$ & $\%$ & \\
\hline Tem outros filhos & & & & & $0,6665^{*}$ \\
\hline Sim & 23 & 39,66 & 95 & 42,79 & \\
\hline Não & 35 & 60,34 & 127 & 57,21 & \\
\hline Ocupação materna & & & & & $0,1075^{*}$ \\
\hline Remunerada & 33 & 56,90 & 100 & 45,05 & \\
\hline Não remunerada & 25 & 43,10 & 122 & 54,95 & \\
\hline Renda familiar & & & & & $0,1727^{* *}$ \\
\hline Menos que 1 salário mínimo & 15 & 25,86 & 38 & 17,12 & \\
\hline de 1 a 2 salários mínimos & 18 & 31,03 & 108 & 48,65 & \\
\hline de 2 a 3 salários mínimos & 16 & 27,59 & 50 & 22,52 & \\
\hline mais que 3 salários mínimos & 7 & 12,07 & 22 & 9,91 & \\
\hline não informou & 2 & 3,45 & 4 & 1,80 & \\
\hline Bolsa família & & & & & $0,9631 *$ \\
\hline Sim & 9 & 15,52 & 35 & 15,77 & \\
\hline Não & 49 & 84,48 & 187 & 84,23 & \\
\hline
\end{tabular}

Fonte: Elaboração própria.

*Teste Qui-quadrado. **Teste G.

Do mesmo modo, 53,2\% das mulheres descreveram a presença de algum sintoma emocional. A Tabela 4 mostra que não houve diferenças estatisticamente significantes entre os fatores epidemiológicos com a probabilidade de problemas e sintomas emocionais para a região em estudo.

Tabela 4 - Relação de fatores epidemiológicos e a presença de sintomas emocionais no período puerperal. Foz do Iguaçu, Paraná, Brasil - 2018 (N=280)

(continua)

\begin{tabular}{|c|c|c|c|c|c|}
\hline \multirow{2}{*}{ Variáveis } & \multicolumn{2}{|c|}{ Sintomas emocionais } & \multicolumn{2}{|c|}{ Sem sintomas emocionais } & \multirow{2}{*}{ Valor de $p$} \\
\hline & $\mathbf{n}$ & $\%$ & $\mathbf{n}$ & $\%$ & \\
\hline \multicolumn{6}{|l|}{ Idade } \\
\hline$\leq 19$ anos & 26 & 17,45 & 19 & 14,50 & $0,7393^{*}$ \\
\hline 20 a 34 anos & 102 & 68,46 & 95 & 72,52 & \\
\hline$\geq 35$ anos & 21 & 14,09 & 17 & 12,98 & \\
\hline \multicolumn{6}{|l|}{ Raça/cor } \\
\hline Branca & 86 & 57,72 & 66 & 50,38 & $0,5479 * *$ \\
\hline Negra & 10 & 6,71 & 12 & 9,16 & \\
\hline Parda & 52 & 34,90 & 51 & 38,93 & \\
\hline Outra & 1 & 0,67 & 1 & 0,76 & \\
\hline Sem registro & 0 & 0,00 & 1 & 0,76 & \\
\hline \multicolumn{6}{|l|}{ Escolaridade } \\
\hline Fundamental completo & 9 & 6,04 & 13 & 9,92 & $0,6394^{* *}$ \\
\hline Fundamental incompleto & 37 & 24,83 & 23 & 17,56 & \\
\hline Médio completo & 57 & 38,26 & 56 & 42,75 & \\
\hline Médio incompleto & 28 & 18,79 & 22 & 16,79 & \\
\hline Superior completo & 1 & 0,67 & 2 & 1,53 & \\
\hline Superior incompleto & 10 & 6,71 & 10 & 7,63 & \\
\hline Sem escolaridade & 7 & 4,70 & 5 & 3,82 & \\
\hline \multicolumn{6}{|l|}{ Situação conjugal } \\
\hline Com companheiro & 132 & 88,59 & 125 & 95,42 & $0,0631^{*}$ \\
\hline Sem companheiro & 17 & 11,41 & 6 & 4,58 & \\
\hline
\end{tabular}


Tabela 4 - Relação de fatores epidemiológicos e a presença de sintomas emocionais no período puerperal. Foz do Iguaçu, Paraná, Brasil - 2018 (N=280)

(conclusão)

\begin{tabular}{|c|c|c|c|c|c|}
\hline \multirow{2}{*}{ Variáveis } & \multicolumn{2}{|c|}{ Sintomas emocionais } & \multicolumn{2}{|c|}{ Sem sintomas emocionais } & \multirow{2}{*}{ Valor de $p$} \\
\hline & $\mathbf{n}$ & $\%$ & $\mathbf{n}$ & $\%$ & \\
\hline \multicolumn{6}{|l|}{ Tem outros filhos } \\
\hline Sim & 65 & 43,62 & 53 & 40,46 & \multirow[t]{2}{*}{$0,5924^{*}$} \\
\hline Não & 84 & 56,38 & 78 & 59,54 & \\
\hline \multicolumn{6}{|l|}{ Ocupação materna } \\
\hline Remunerada & 70 & 46,98 & 63 & 48,09 & \multirow[t]{2}{*}{$0,8525^{*}$} \\
\hline Não remunerada & 79 & 53,02 & 68 & 51,91 & \\
\hline \multicolumn{6}{|l|}{ Renda familiar } \\
\hline $\begin{array}{l}\text { Menos que } 1 \text { salário } \\
\text { mínimo }\end{array}$ & 33 & 22,15 & 20 & 15,27 & \multirow[t]{5}{*}{$0,6615^{* * *}$} \\
\hline 1 a 2 salários mínimos & 64 & 42,95 & 62 & 47,33 & \\
\hline 2 a 3 salários mínimos & 33 & 22,15 & 33 & 25,19 & \\
\hline $\begin{array}{l}\text { mais que } 3 \text { salários } \\
\text { mínimos }\end{array}$ & 16 & 10,74 & 13 & 9,92 & \\
\hline não informou & 3 & 2,01 & 3 & 2,29 & \\
\hline \multicolumn{6}{|l|}{ Bolsa família } \\
\hline Sim & 23 & 15,44 & 21 & 16,03 & \multirow[t]{2}{*}{$0,9775^{*}$} \\
\hline Não & 126 & 84,56 & 110 & 83,97 & \\
\hline
\end{tabular}

Fonte: Elaboração própria.

*Teste Qui-quadrado. **Teste G.

Embora esses resultados não tenham mostrado significância estatística, os sintomas emocionais estiveram mais presentes para mulheres com os extremos de idade, brancas, sem companheiro e com profissão não remunerada. Muitas destas mulheres descreveram condições emocionais desfavoráveis, sendo que aproximadamente $20 \%$ relataram estar nervosas e 30\% informaram sintomas emocionais, que podem fragilizar o processo da maternidade, conforme apresenta o Gráfico 1. 
Gráfico 1 - Condições e sintomas emocionais apresentados por mulheres no período puerperal. Foz do Iguaçu, Paraná, Brasil - 2018 (N=280)

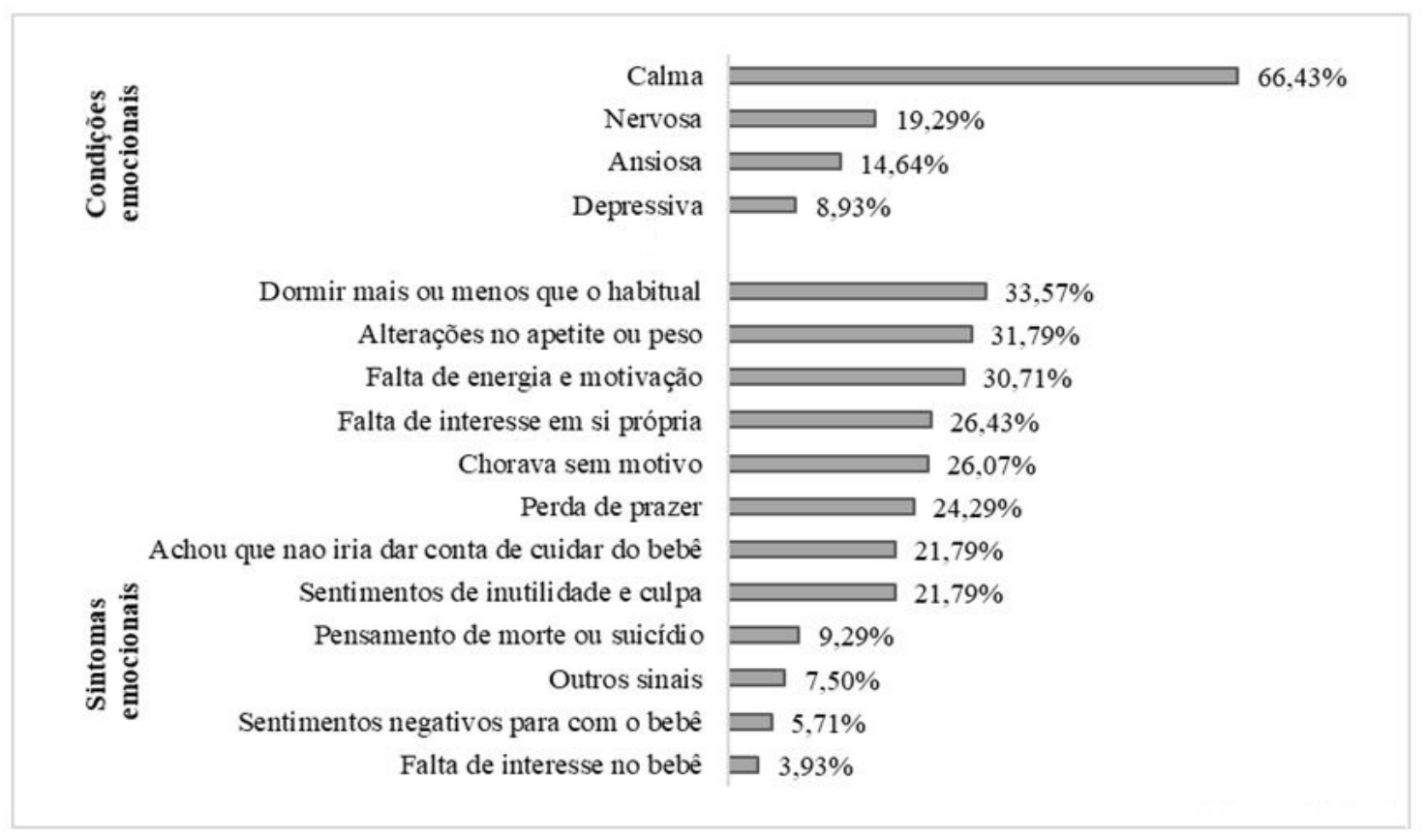

Fonte: Elaboração própria.

Importante destacar que os sintomas emocionais descritos pelas puérperas envolveram desde sentimentos sobre o cuidado de si, como também sobre o cuidado do bebê em domicílio. Foi identificado que cerca de 10\% das puérperas participantes apresentaram sintomas depressivos e pensamentos suicidas.

\section{Discussão}

Os resultados mostraram que apenas a metade das mulheres participantes realizou a consulta puerperal na primeira semana após o parto na região estudada. Segundo a RMP, os profissionais atuantes nos serviços de Atenção Primária à Saúde (APS) devem realizar uma visita domiciliar na primeira semana após o parto e o nascimento (até o 5o dia), para acompanhamento da saúde da puérpera e da criança, assim como deverá ser garantido uma consulta no puerpério, na primeira semana pós-parto. A razão para a realização desse acompanhamento precoce se refere aos altos índices de morbidades maternas e mortalidade infantil no período neonatal precoce, isto é, nos primeiros seis dias de vida ${ }^{(8)}$.
Outro aspecto a destacar refere-se à adesão das mulheres para a consulta puerperal no prazo de 42 dias, ou ainda estendido para até 90 dias, o qual não atingiu sua totalidade. Fatores como a baixa idade e escolaridade estiveram presentes entre as mulheres que não aderiram à consulta puerperal neste estudo. Segundo pesquisadores, a gravidez precoce e a falta de seguimento à saúde nessas situações, podem se relacionar com classes econômicas menos favorecidas, desemprego, inserção precoce no mercado de trabalho informal, desistência escolar e desafios conjugais $^{(4-5)}$, tendo em vista que há dificuldades para que esta mulher jovem e com baixa escolaridade, compreenda a necessidade e a importância de continuar em acompanhamento, mesmo sem apresentar problemas de saúde.

Além desses fatores para a falta de adesão ao seguimento puerperal, um estudo de revisão realizado por pesquisadores da Bahia indicou que embora a assistência profissional no período puerperal seja fundamental para potencializar a saúde materna e infantil, na prática, observa-se que este atendimento é pouco valorizado pelas mulheres, tendo em vista que após o parto, a 
mãe mantém o olhar direcionado apenas para a saúde do filho, não realizando o seguimento à sua saúde, e, em geral, não participam das consultas puerperais ${ }^{(10)}$. O mesmo fenômeno ocorreu no cenário em estudo, apesar de uma política própria para saúde materno-infantil no Paraná.

Ademais, as práticas educativas desenvolvidas por profissionais de saúde nos serviços de atenção primária ainda permanecem atreladas às tradicionais palestras, com predomínio da prática pedagógica tradicional, utilizando-se recursos precários e linguagem acessível, porém, pouco interativa e que resulta na baixa participação dos usuários $^{(11)}$, principalmente quando se trata de uma população mais jovem e com baixa escolaridade, como encontrado no presente estudo.

Com respeito aos agravos à saúde no período puerperal, identificou-se que cerca de $20 \%$ das mulheres participantes apresentaram problemas clínicos, corroborando outros estudos que mostraram que os principais problemas maternos nesse período incluem infecções, dores, hipertensão arterial, ingurgitamento mamário, mastite, desmame precoce, entre outros ${ }^{(12-14)}$. Tendo em vista que os problemas envolvem desde agravos mais simples, como também situações que requerem maior atenção, destaca-se que a assistência puerperal, em especial no ambiente domiciliar, possibilitará identificar e intervir diretamente nesses problemas cotidianos, minimizando agravos e contribuindo para a promoção da saúde ${ }^{(15)}$. A ausência de agravos à saúde leva muitas mulheres a acreditarem não ser necessária a realização do seguimento puerperal.

Um estudo brasileiro mostrou que a presença de complicações puerperais tem ampla relação com a atenção pré-natal e com a realização de cesarianas. Com base nesses achados, os autores destacaram a necessidade de aprimorar a atenção à saúde das gestantes, ampliando-se o número de consultas pré-natal, assim como incentivando a valorização do parto normal, com vistas a reduzir a morbimortalidade materna, em especial no período puerperal ${ }^{(16)}$.

Somado aos problemas físicos, identificou-se que mais de $50 \%$ das mulheres apresentaram problemas ou sintomas emocionais no pós-parto. Embora não tenha identificado significância estatística, os extremos de idade, o fato de não ter um companheiro e ser baixa renda, foram aspectos que se mostraram como preditores de sintomas emocionais.

Um estudo com mulheres inglesas mostrou que a fadiga pós-parto não é inevitável e pode afetar uma proporção substancial de mulheres, sendo que a idade e a paridade são preditores para o aparecimento de sintomas emocionais, mas a ajuda prática e o apoio dos parceiros podem funcionar como fatores de proteção ${ }^{(5)}$. Já outro estudo com americanas mostrou que $63 \%$ de mulheres de baixa renda podem apresentar sintomas emocionais entre 1 e 3 meses de pós-parto ${ }^{(17-18)}$.

Aspecto evidenciado por outros estudos para o surgimento da fadiga emocional no período puerperal foi a presença de problemas clínicos como anemia, infecção e hemorragia, assim como o baixo nível socioeconômico, desemprego, primiparidade, e maior idade materna, embora também não tenham mostrado significância estatística nos estudos ${ }^{(17,19-21)}$. Inclusive, outro estudo americano identificou a relação entre a situação socioeconômica desfavorável com a piora do padrão do sono e, consequentemente, sintomas emocionais e fadiga materna no período puerperal ${ }^{(22)}$.

Um estudo brasileiro destacou que o acompanhamento puerperal sobressai a identificação de sinais e sintomas que levam a riscos de saúde física, pois este é um período oportuno para identificar adversidades às quais as famílias estão expostas, como por exemplo, as dificuldades financeiras, que, muitas vezes, está atrelada ao risco de não alcançar as condições saudáveis de vida, significativamente presente entre as famílias de países em desenvolvimento como o Brasil. Nessas dimensões, a atuação profissional estende-se para além dos fatores biológicos, favorecida pela estratégia de realização da visita domiciliar, importante ferramenta para a promoção e vigilância em saúde ${ }^{(23)}$.

No tocante aos sinais e sintomas apresentados pelas mulheres após seis meses de nascimento 
do filho, verifica-se que os sintomas mais cotidianos, como falta de sono, apetite alterado, vontade de chorar e falta de motivação foram os mais encontrados, contudo, sintomas mais intensos como a depressão e os pensamentos suicidas estiveram presentes para aproximadamente $10 \%$ das participantes.

Pesquisas mostram que a maioria das mulheres experiencia algum tipo de distúrbio mental no pós-parto, com alterações no humor e depressão leve ${ }^{(5,24)}$. No entanto, algumas mulheres podem apresentar sintomas mais graves, desencadeando a depressão grave ou até mesmo a psicose. Importante destacar que sintomas mais leves, apresentados de forma contínua, podem levar a uma sobrecarga materna, e consequentemente, a sintomas mais graves ${ }^{(5)}$.

A psicose pós-parto se refere a uma forma grave de distúrbio mental que se caracteriza por extrema confusão, perda de contato com a realidade, paranoia, delírios, processos de pensamento desorganizados e alucinações ${ }^{(25)}$. Pode afetar cerca de duas a cada mil mulheres em idade fértil e ocorre nas seis primeiras semanas após o parto. Embora seja relativamente rara, é vista como uma emergência psiquiátrica considerando o risco iminente de suicídio ou infanticídio ${ }^{(5)}$.

Tendo em vista que muitas mulheres apresentam condições emocionais desfavoráveis no período puerperal e requerem cuidados adicionais, estudos indicam como estratégia de intervenção a autogestão, suporte por telefone ou por visitas domiciliares, exercícios e tecnologias educacionais em saúde ${ }^{(22,25)}$. Além disso, apoio dirigido e disponibilidade para aconselhamento para atender as necessidades singulares de cada puérpera devem ser encorajados entre os profissionais e serviços de saúde. É preciso ressaltar que situações adversas no processo de nascimento, a exemplo da prematuridade, podem representar fatores determinantes para o sofrimento emocional materno, exigindo um preparo da família para o enfrentamento e transição da parentalidade ${ }^{(5)}$.

Como limitações do presente estudo, aponta-se para a necessidade de dar voz às puérperas e aos profissionais de saúde dos serviços de atenção primária para que possam apontar os fatores determinantes para contemplar a integralidade das ações em saúde no período puerperal.

\section{Conclusão}

Os resultados apontaram a adesão insatisfatória de consultas puerperais, tanto para a consulta na primeira semana após o parto quanto para a consulta até 42 dias após o parto, e identificou a relação entre a não realização das consultas aos 42 dias de puerpério com a baixa idade materna e baixa escolaridade. Problemas clínicos no período pós-parto estiveram presentes para aproximadamente 20\% das mulheres, e sintomas emocionais, descritos por mais da metade das participantes, mostraram-se preocupantes para o cenário em estudo.

Conclui-se que fatores epidemiológicos têm influência para a adesão insatisfatória de consultas puerperais, mas não para o aparecimento de problemas de saúde no puerpério.

Desse modo, é preciso enfatizar a necessidade de estratégias para ampliar a adesão às consultas no período pós-parto, e assim, atender integralmente a mulher no puerpério, adotando-se ferramentas importantes como a visita domiciliar e as tecnologias educacionais para atender de forma plena a saúde materna no pós-parto, criando-se oportunidades de proporcionar ações que visem a promoção da saúde e a prevenção de agravos em dimensões individuais e coletivas.

Não obstante a existência de políticas governamentais para atenção à saúde materna no puerpério, como a proposta da RMP, há necessidade de ampliar essas ações e de fato colocá-las em prática, com vistas a atender as reais necessidades das famílias.

\section{Fontes de financiamento:}

Conselho Nacional de Desenvolvimento Científico e Tecnológico (CNPq).

\section{Colaborações:}

1 - concepção, projeto, análise e interpretação dos dados: Isabela Cristina Beskow 
12

Influência de fatores epidemiológicos no seguimento e aparecimento de problemas puerperais

Oliveira, Rosane Meire Munhak da Silva, Helder Ferreira, Rosangela Aparecida Pimenta Ferrari e Adriana Zilly;

2 - redação do artigo e revisão crítica relevante do conteúdo intelectual: Rosane Meire Munhak da Silva, Rosangela Aparecida Pimenta Ferrari e Adriana Zilly;

3 - aprovação final da versão a ser publicada: Isabela Cristina Beskow Oliveira, Rosane Meire Munhak da Silva, Helder Ferreira, Rosangela Aparecida Pimenta Ferrari e Adriana Zilly.

\section{Referências}

1. Corrêa MSM, Feliciano KVO, Pedrosa EN, Souza AI. Acolhimento no cuidado à saúde da mulher no puerpério. Cad saúde pública. 2017:33(3):e00136215. DOI: 10.1590/0102-311X0 0136215

2. Mazzo MHSN, Brito RS. Instrumento para consulta de enfermagem à puérpera na atenção básica. Rev bras enferm. 2016;69(2):294-303. DOI: http:// dx.doi.org/10.1590/0034-7167.2016690215i

3. Medeiros LS, Costa ACM. Período puerperal: a importância da visita domiciliar para enfermeiros da Atenção Primária à Saúde. Rev Rene [Internet]. 2016 [cited 2019 Oct 10]; 17(1):112-9. Available from: http://periodicos.ufc.br/rene/article/view/2622

4. Miranda DB, Marostica FC, Matão MEL. Influência do fator cultural no processo de cuidado puerperal. Rev eletr gestão saúde [Internet]. 2015 [cited 2019 Oct 8];6(3):2444-59. Available from: https://dialnet.unirioja.es/servlet/articulo? codigo $=5555805$

5. Henderson J, Alderdice F, Redshaw M. Factors associated with maternal postpartum fatigue: an observation al study. BMJ Open. 2019;9(7):e025927. DOI: http://dx.doi.org/10.1136/ bmjopen-2018-025927

6. Johnson PD, Duzyj CM, Howell EA, Janevic T. Patient and hospital characteristics associated with severe maternal morbidity among postpartum readmissions. J Perinatol. 2019;39(9):1204-12. DOI: https://doi.org/10.1038/s41372-019-0426-6

7. Brasil. Ministério da Saúde. Cadernos de Atenção Básica: atenção ao pré-natal de baixo-risco [Internet]. Brasília (DF); 2012 [cited 2019 Oct 10]. Available from: https://bvsms.saude.gov.br/bvs/ publicacoes/cadernos_atencao_basica_32_ prenatal.pdf
8. Paraná. Secretaria de Estado da Saúde do Paraná. Linha guia: Rede Mãe Paranaense [Internet]. 7 a ed. Curitiba 2018 [cited 2019 Oct 10]. Available from: http://www.saude.pr.gov.br/arquivos/File/ LinhaGuiaMaeParanaense_2018.pdf

9. Pito ALBS. Epidemiologia aplicada nos serviços de saúde. São Paulo: Martinari; 2012.

10. Gomes GF, Santos APV. Assistência de enfermagem no puerpério. Rev enferm contemp. 2017;6(2):211-20. DOI: $10.17267 / 2317-3378$ rec. v6i2.1407

11. Guerreiro EM, Rodrigues DP, Queiroz ABA, Ferreira MA. Educação em saúde no ciclo gravídico-puerperal: sentidos atribuídos por puérperas. Rev bras enferm. 2014;67(1):13-21. DOI: 10.5935/0034-7167.20140001

12. Frota MA, Lopes MF, Lima KF, Sales COCB, Silva CAB. Interfaces of the discontinuation of breastfeeding. Acta sci, Health sci. 2016;38(1):33-8. DOI: https://doi.org/10.4025/actascihealthsci.v38i1. 28514

13. Monteiro TLVA, Silva RC, Sousa GC, Neiva MJLM. Eventos de infecção puerperal em uma maternidade de referência no município de Caxias, Maranhão. Rev enferm UFPI [Internet]. 2016 [cited 2019 Sep 29];5(2):11-15. Available from: https://revistas.ufpi.br/index.php/reufpi/article/ view/5110/pdf

14. Skupien SV, Ravelli APX, Acauan LV. Consulta puerperal de enfermagem: prevenção de complicações mamárias. Cogitare enferm [Internet]. 2016 [cited 2019 Oct 1];21(2):1-6. Available from: http://www.redalyc.org/jatsRepo/4836/ 483653650019/483653650019.pdf

15. Rocha GM, Cordeiro RC. Assistência domiciliar puerperal de enfermagem na estratégia saúde da família: intervenção precoce para promoção da saúde. Rev Uni Vale Rio Verde. 2015;13(2):483-93. DOI: http://dx.doi.org/10.5892/ruvrd.v13i1.2345

16. Lima THB, Amorim MM, Buainain Kassar S, Katz L. Maternal near miss determinants at a maternity hospital for high-risk pregnancy in northeastern Brazil: a prospective study. BMC Pregnancy Childbirth. 2019;19(1):271. DOI: https:// doi.org/10.1186/s12884-019-2381-9

17. Bakker $M$, van der Beek AJ, Hendriksen IJM, Bruinvels DJ, van Poppel MN. Predictive factors of postpartum fatigue: a prospective cohort study among workingwomen. J Psychosom Res. 2014;77(5):385-90. DOI: https://doi.org/10.1016/j. jpsychores.2014.08.013 
18. Doering JJ, Sims DA, Miller DD. How postpartum women with depressive symptoms manage sleep disruption and fatigue. Res nurs health. 2017;40(2):132-42. DOI: https://doi.org/10.1002/ nur. 21782

19. Van Der Woude D, Pijnenborg JMA, Verzijl JM, Van Wijk EM, De Vries J. Health status and fatigue of postpartum anemic women: a prospective cohort study. Eur J Obst Gynecol Reprod Biol. 2014;181:119-23. DOI: https://doi.org/10.1016/j. ejogrb.2014.07.028

20. Lai YL, Hung CH, Stocker J, Chan TF, Liu Y. Postpartum fatigue, baby-care activities, and maternal-infant attachment of vaginal and cesarean births following rooming-in. Appl nurs res. 2015;28(2):116-20. DOI: https://doi.org/10.1016/j. apnr.2014.08.002

21. Mori E, Tsuchiya $M$, Maehara $K$, Iwata $H$, Sakajo A, Tamakoshi K. Fatigue, depression, maternal confidence, and maternal satisfaction during the first month postpartum: a comparison of Japanese mothers by age and parity. Int $\mathrm{J}$ Nurs Prac. 2017;23(1):e12508. DOI: https://doi. org/10.1111/ijn.12508
22. Doering JJ, Dogan S. A postpartum sleep and fatigue intervention feasibility pilot study. Behav Sleep Med. 2018;16(2):185-201. DOI: https://doi. org/10.1080/15402002.2016.1180523

23. Andrade RD, Santos JS, Maia MAC, Mello DF. Fatores relacionados à saúde da mulher no puerpério e repercussões na saúde da criança. Esc Anna Nery. 2015;19(1):181-6. DOI: http://dx.doi. org/10.5935/1414-8145.20150025

24. Giallo R, Gartland D, Woolhouse H, Brown S. Differentiating maternal fatigue and depressive symptoms at six months and four years postpartum: considerations for assessment, diagnosis and intervention. Midwifery. 2015;31(2):316-22. DOI: https://doi.org/10.1016/j. midw.2014.09.005

25. Teixeira E, Martins TDR, Miranda PO, Cabral BG, Silva BAC, Rodrigues LSS. Tecnologia educacional sobre cuidados no pós-parto: construção e validação. Rev baiana enferm. 2016;30(2):1-10. DOI: http://dx.doi.org/10.18471/rbe.v30i2.15358

Recebido: 6 de março de 2020

Aprovado: 3 de abril de 2020

Publicado: 28 de abril de 2020

A Revista Baiana de Enfermagem utiliza a Licença Creative Commons - Atribuição-NãoComercial 4.0 Internacional. https://creativecommons.org/licenses/by-nc/4.0/ Este artigo é de acesso aberto distribuído sob os termos da Licença Creative Commons (CC BY-NC). Esta licença permite que outros remixem, adaptem e criem a partir do seu trabalho para fins não comerciais. Embora os novos trabalhos tenham de lhe atribuir o devido crédito e não possam ser usados para fins comerciais, os usuários não têm de licenciar esses trabalhos derivados sob os mesmos termos. 